\title{
BMJ Open Characterising Arm Recovery in People with Severe Stroke (CARPSS): protocol for a 12-month observational study of clinical, neuroimaging and neurophysiological biomarkers
}

\author{
Kathryn S Hayward, ${ }^{1,2,3}$ Keith R Lohse, ${ }^{4}$ Julie Bernhardt, ${ }^{2,3}$ Catherine E Lang, ${ }^{5}$ \\ Lara A Boyd ${ }^{1}$
}

To cite: Hayward KS,

Lohse KR, Bernhardt J, et al. Characterising Arm Recovery in People with Severe Stroke (CARPSS): protocol for a 12-month observational study of clinical, neuroimaging and neurophysiological biomarkers. BMJ Open 2018;8:e026435. doi:10.1136/ bmjopen-2018-026435

- Prepublication history for this paper is available online. To view these files, please visit the journal online (http://dx.doi. org/10.1136/bmjopen-2018026435).

Received 7 September 2018 Revised 3 October 2018 Accepted 5 October 2018
Check for updates

(C) Author(s) (or their employer(s)) 2018. Re-use permitted under CC BY-NC. No commercial re-use. See rights and permissions. Published by BMJ.

For numbered affiliations see end of article.

Correspondence to Dr Kathryn S Hayward; kathryn.hayward@ubc.ca

\section{ABSTRACT}

Introduction In individuals with early (indexed $\leq 7$ days poststroke) and severe upper limb paresis (shoulder abduction and finger extension score of $<5$ out of 10 ), our objectives are to: (1) determine if biomarkers of brain structure and function collected at $<1$ month poststroke explain who will experience clinically important recovery over the first 12 months poststroke; (2) compare stroke survivors' perceptions of personally meaningful recovery to clinically important recovery; and (3) characterise the trajectory of change in measures of motor function, brain structure and function.

Methods and analysis Prospective observational study with an inception cohort of 78 first-time stroke survivors. Participants will be recruited from a single, large tertiary stroke referral centre. Clinical and biomarker assessments will be completed at four follow-up time points: 2 to 4 weeks and 3,6 and 12 months poststroke. Our primary outcome is achievement of clinically important improvement on two out of three measures that span impairment (Fugl-Meyer Upper Limb, change $\geq 10$ points), activity (Motor Assessment Scale item 6, change $\geq 1$ point) and participation (Rating of Everyday Arm-use in the Community and Home, change $\geq 1$ point). Brain biomarkers of structure and function will be indexed using transcranial magnetic stimulation and MRI. Multilevel modelling will be performed to examine the relationship between clinically important recovery achieved (yes/no) and a priori defined brain biomarkers related to the corticospinal tract and corpus callosum. Secondary analyses will compare stroke survivor's perception of recovery, as well as real-world arm use via accelerometry, to the proposed metric of clinically meaningful recovery; and model trajectory of recovery across clinical, a priori defined biomarkers and exploratory variables related to functional connectivity. Ethics and dissemination Approved by the hospital and university ethics review boards. Results will be disseminated through peer-reviewed publications and conference presentations.

Trial registration number NCT02464085.

\section{INTRODUCTION}

Up to $30 \%$ of acute stroke patients have severe upper limb impairment. ${ }^{1}$ Clinical staff find it

\section{Strengths and limitations of the study}

- Focused investigation of a neglected subgroup of stroke survivors with early, severe upper limb paresis.

- Clinical measures span the domains of the International Classification of Functioning, Disability and Health, and biomarkers span structural and functional methods.

- Data collection occurs at biologically relevant time points across the first year poststroke.

- Builds knowledge across the spectrum of clinical, biological and survivor perceptions of meaningful recovery.

- Findings from this single-centre inception cohort have the potential to inform clinical trial design elements of stratification and intervention timing.

challenging to answer questions from patients such as 'how much recovery might I expect to get back in my arm, how long will it take for me to recover, and will my recovery be meaningful?' Since people with early and severe impairment have a 50:50 chance of recovering some function versus not recovering, ${ }^{2}$ and clinical measures have been found to add little to the ability to predict expected recovery, ${ }^{3}$ answering these questions is difficult. The inadequacy of current clinical models to explain expected recovery was highlighted in the first international Stroke Rehabilitation and Recovery Roundtable biomarker consensus statement ${ }^{4}$ in which the need to develop and use biomarkers that relate to neurobiology and mechanisms of recovery to stratify or subgroup research participants in trials was a strong recommendation.

The most promising potential biomarkers for stratification of people with upper limb impairment (mild through to severe) relate 
to the structure and function of the corticospinal tract (CST). The presence or absence of a motor-evoked potential (MEP+, MEP-) indexed using transcranial magnetic stimulation (TMS) captured from electromyography (EMG) of forearm musculature early after stroke (within 7-10 days) explains initial Fugl-Meyer Upper Limb (FM-UL) outcome ${ }^{5}$ and predicts expected recovery. ${ }^{6}$ This biomarker is largely influenced by the structural integrity and function of the CST. However, this dichotomised outcome (MEP+, MEP-) may not be sufficiently sensitive to meaningfully measure differences in the subgroup of individuals with severe upper limb impairment.

Here, we define severe upper limb impairment by the presence of early paresis of shoulder abduction and finger extension (SAFE) manual muscle test combined score of $<5$ out of a possible 10 points, which indicates little to no antigravity movement is possible. Our individual patient data review ${ }^{5}$ found that while MEP+ status was associated with better motor outcome in the severe subgroup, there was considerable variability in outcome scores (FM-UL) within the MEP+ and MEP- cohorts. Other studies have demonstrated that $15 \%-20 \%$ of individuals who are MEP - go on to experience some recovery. ${ }^{7-9}$

The contribution of other possible biomarkers that index CST have largely focused on neuroimaging methods. Information can be captured using anatomical scans (T1) to identify overlap between the lesion and a mask of the $\mathrm{CST}^{10}{ }^{10}$ and diffusion tensor imaging to index (1) CST microstructural properties (eg, fractional anisotropy (FA) to understand directionality), (2) FA asymmetry index (CST AI) between lesioned and unlesioned hemisphere ${ }^{11}$ or (3) tract number. ${ }^{12}$ These structural approaches concentrate on the impact of the lesion on the CST. Like MEP status, they are important when considering the spectrum of mild through to severe impairment. ${ }^{13}$ However, when focused on the subgroup with severe impairment, CST AI is not related to impairment. ${ }^{513}$ This suggests that other factors may be important to explain expected recovery in the severe subgroup.

Inclusion of biomarkers beyond the CST may lead to enhanced models of recovery in individuals with severe motor impairment. For instance, corpus callosum (CC) white matter integrity using diffusion imaging appears to explain almost $50 \%$ of the variance in FM-UL outcome in this group. ${ }^{14}$ Other work has focused on the inter-relationships between the CST and other motor pathways using diffusion imaging, including the reticulospinal and premotor pathways to effect motor outcomes. ${ }^{15}$ Collectively, research into such alternative pathways has largely included people with chronic impairment ( $>6$ months poststroke); therefore, it is unknown if such regions are important early poststroke or represent compensatory regions relevant in the chronic phase.

Biomarkers of functional neuroimaging have received the least attention. ${ }^{4}$ A potential biomarker in the severe cohort is brain network activity measured with resting state functional MRI. As this approach can be used in patients with little to no movement poststroke to interrogate all brain networks simultaneously, it was defined as a developmental priority by the Stroke Rehabilitation and Recovery Roundtable consensus group. ${ }^{4}$ Consistent with structural neuroimaging data that highlight the importance of the CC, resting state functional connectivity in the early and late subacute phases shows that interhemispheric connectivity is of particular importance to motor recovery. ${ }^{10}$

Taken together, past work suggests that upper limb biomarkers are not one-size-fits-all. ${ }^{16}$ In the current paper, we detail the protocol for a longitudinal study that aims to link the neurobiology of human stroke to the observed trajectory of motor recovery, advancing current knowledge of upper limb recovery in people with severe impairment. Importantly, we will link our neurobiological and clinical findings to stroke survivor perspectives of what $\mathrm{s} /$ he considers to be meaningful recovery. This has not been addressed in any prior biomarker work. The findings of the current study can ultimately help determine the 'right person' to target and 'right time' for therapeutic intervention, as well as enabling more precise patient stratification of people with severe upper limb impairment poststroke in future trials.

The primary aim is to identify biomarker(s) of brain structure and/or function that explain which individuals with severe upper limb paresis in the first 7 days poststroke will go on to experience clinically important recovery over the first 12 months poststroke. We hypothesise that the $\mathrm{CC}$ will explain unique variance in recovery above and beyond the CST. Based on our prior work, ${ }^{51314}$ we expect the CST itself to explain negligible variation in people with severe impairment.

Secondary aims are to:

1. Describe, from the stroke survivor perspective, what is meaningful recovery, and understand when it is achieved over the first 12 months poststroke. We will also consider factors that might contribute to achievement, and how survivors' perceptions of meaningful recovery relate to our defined metric of clinically important recovery. We hypothesise that biomarker-defined clinically important subgroups, will not be consistent with stroke survivors' perceptions of personally meaningful recovery.

2. Characterise the trajectory of motor recovery across the International Classification for Functioning, Disability and Health (ICF) domains of impairment, activity, participation and quality of life over the first 12 months poststroke. We hypothesise that most recovery will occur early rather than later poststroke, but there will be a subgroup of late recoverers.

3. Establish how brain structure and function change over the first 12 months poststroke using neuroimaging and neurophysiological measures. We hypothesise that structural biomarkers will have a relatively flat trajectory throughout the first 12 months poststroke; however, functional biomarkers will show stronger trajectories that are associated with motor recovery. 


\section{METHODS}

\section{Design}

A prospective observational study of an inception cohort of individuals with severe upper limb paresis indexed $\leq 7$ days poststroke (baseline) and followed up at four time points over the first 12 months poststroke. Follow-up time points (+/-2weeks) are both clinically and biologically relevant:

- 2-4 weeks, stabilisation of acute changes ${ }^{17}$;

- 3 months, heightened window of plasticity presumed to $\operatorname{taper}^{18}$ and reflect end of early subacute recovery phase $^{19}$;

- 6 months, end of late subacute recovery phase $^{19}$; and

- 12 months, functional stabilisation. ${ }^{20}$

\section{Participants and study sites}

Patients who had a stroke and are admitted to a tertiary stroke referral centre in British Columbia Canada will be screened for eligibility. Approximately 600 patients with stroke are admitted annually. Stroke will be confirmed by medical practitioner diagnosis using CT or MRI scans at the recruiting centre. Patients deemed potentially eligible will continue to be screened until they either meet eligibility criteria, or are ineligible. Participants will be followed at two rehabilitation centres in Vancouver British Columbia Canada; all participants outside these catchment centres will be ineligible.

Patients will be eligible to participate if they are an adult (aged 18 to 90 years old) with severe upper limb paresis (SAFE score $<5 / 10$ ) $\leq 7$ days following first clinically diagnosed stroke (ischaemic or haemorrhagic). Exclusion criteria include another neurological condition, psychiatric illness, other medical condition(s) deemed to significantly limit function, palliative medical management, insufficient communication and/or no access to an appropriate substitute decision maker (eg, carer, family) or interpreter.

\section{Recruitment}

Patients deemed to fulfil all eligibility criteria will be invited to participate. Informed consent will be sought from the eligible patient or, if unable to provide written informed consent, an appropriate substitute decision maker will be approached to provide written consent. If English is their second language, we will ensure an appropriate translator or substitute decision maker is available. Once the patient gives informed consent, screening for TMS and MRI testing will be completed, demographic and baseline data collected and appropriate testing organised. Contraindication to either TMS or MRI precludes a patient from participating in the specific testing, but not the overall study. Demographic information (including age, sex, comorbidities) and stroke details (including stroke location, lesion type, use of thrombolysis/thrombectomy, admission and 24-hour National Institutes of Health Stroke Scale (NIHSS)) will be collated from the admission record or through patient interview, ${ }^{21}$ and participants will be asked to give consent to access routine clinical scan reports (CT and MRI).

\section{Outcomes}

Dependent variable: clinically important recovery

The dependent variable is clinically important recovery. To date, there is no defined metric of clinically important recovery, nor a metric that encapsulates all ICF domains to describe recovery within a single outcome. Therefore, for the purposes of this study, we define clinically important recovery as change of at least $15 \%-20 \%$ of the scale across two out of three upper limb measures that span ICF domains. ${ }^{22}$ The moment when a participant hits this benchmark on ANY two of these measures, they will be categorised as achieving clinically important change. The indices are:

- Impairment using the FM-UL: test comprises 33 items rated from 0 to 2 , where $0=$ cannot perform, $1=$ performs partially, 2=performsfully, totalling 66 points. This measure has good psychometric properties $^{23}$ and has been recommended as a core data element by the Stroke Rehabilitation and Recovery Roundtable. ${ }^{21}$ A meaningful change for this outcome is defined as $\geq 10$ points ( $15 \%$ change), which is consistent with past work of a clinically important change in motor impairment in the early stages of recovery. ${ }^{24}$

- Activity limitations using the Motor Assessment Scale items 6,7 and 8 (MAS-UL): each item is rated out of a possible six points, leading to a possible 18 points in total. MAS6 measures upper arm function, MAS7 measures hand movements and MAS8 measures advanced hand movements. This measure has good psychometric properties. ${ }^{25}$ A meaningful change is undefined, but it has been previously demonstrated that $\geq 1$ point ( $15 \%$ change) on MAS6 reflects a shift in upper limb function ability. ${ }^{26}$ For the combined MAS-UL used in this study, a change of $\geq 3$ points is required.

- Participation restriction of use of the arm in everyday life will be indexed using the Rating of Everyday Arm-use in the Community and Home (REACH): self-report measure of real-world task arm use completed via interview ${ }^{27}$ and rated out of a possible five points. Importantly, REACH indexes whether the stroke affected arm is a participant's dominant or non-dominant arm. Meaningful change remains undefined on this measure, but in line with previous work, we adopted $\geq 1$ point ( $15 \%$ ) change.

\section{Structural and functional brain measures}

TMS will be used to characterise motor cortex excitability at rest and when active for the ipsilesional $\mathrm{CST}^{28}$ using a Magstim $200^{2}$ unit with a figure-8 coil. We will test a distal (extensor carpi radialis) and proximal (lateral head of triceps) muscle using standard protocols. ${ }^{28}{ }^{29}$ Resting motor threshold will be defined as the percentage of stimulator output intensity (\% MSO) that elicits a MEP $>50 \mu \mathrm{V}$ in 5 of 10 trials. Active motor threshold is the \% MSO intensity that elicits a MEP $>200 \mu \mathrm{V}$ in 5 of 10 trials while the participant holds a resisted contraction consistent with 
the muscle of interest (wrist extension or elbow extension). Intensity of active muscle activity will be monitored real-time via EMG to ensure consistency. Breaks will be provided every 3-4 trials to ensure that fatigue does not influence performance. A trained therapist will provide resistance for all active movements. Participants will be characterised as MEP present (MEP+) or absent (MEP-) for both resting and active motor threshold testing of each muscle of interest. If a participant is MEP+, we will complete a recruitment curve $(100 \%, 110 \%, 120 \%$, $130 \%, 140 \%, 150 \%)$ as able. If a participant is MEP-, we will characterise the number of trials a MEP was present per above criteria (out of 10) to afford serial probing in this cohort.

A MRI scan will be performed at 2-4 weeks and 3, 6 and 12 months on a GE Discovery MR750 3T scanner using a 32-channel head coil. We will collect both structural and functional sequences:

1. T1: $256 \mathrm{FOV}, 256 \times 256$ matrix, $1 \mathrm{~mm}$ slices, interleaved bottom up, scan duration $\sim 5$ min.

2. T2 Fluid-attenuated inversion recovery (FLAIR): TR $9000 \mathrm{~ms}$, TE $140 \mathrm{~ms}$, TE2 $90 \mathrm{~ms}, 256 \mathrm{FOV}, 256 \times 256 \mathrm{ma}-$ trix, $3 \mathrm{~mm}$ slices, interleaved bottom up, scan duration $\sim 5 \mathrm{~min}$.

3. Diffusion tensor imaging (High Angular Resolution Diffusion Imaging (HARDI)): TR $7000 \mathrm{~ms}$, TE minimum, 73 slices, $2 \mathrm{~mm}$ thick, interleaved bottom up (60 directions, $b=1000$ ), 3B0 interspersed throughout the sequence, scan duration $\sim 7 \mathrm{~min}$.

4. Resting state functional MRI: TR $2000 \mathrm{~ms}$, TE $30 \mathrm{~ms}$, 150 samples, $3 \mathrm{~mm}$ slices with $1 \mathrm{~mm}$ spacing, interleaved bottom up, two scan runs at 5 min each.

We will use the T1 and FLAIR scans in MRICRON to hand draw the stroke lesion and create a mask. Diffusion tensor imaging will be processed in FMRIB Software Library (FSL) and hand drawn regions of interest will be placed around the CST (using landmarks of the base of the corona radiata and anterior commissure ${ }^{30}$ ), and the CC (segmented into five regions ${ }^{31}$ ). Resting state sequences will be analysed to identify connectivity patterns.

\section{Other outcomes}

All measures will be collected at every follow-up time point, that is, 2-4 weeks and 3, 6 and 12 months poststroke. See table 1 for a list of outcomes and time points of collection.

Arm use will be measured using ActiGraph 3GTx accelerometers (ActiGraph, Florida, USA) that are worn for 7 consecutive days to quantify real-world arm use. Participants will be fitted with two accelerometers: less affected wrist and more affected wrist at each follow-up time point. Accelerometry data will be analysed using ActiLife software. Subsequent custom-built Matlab scripts will be used to describe mean upper limb use per day (hours), ratio of arm use per day (more affected: less affected hours), intensity of bilateral upper limb activity (bilateral magnitude) and the contribution of both upper limbs to activity (magnitude ratio) for each second of activity per day. ${ }^{32}$ Density plots will be used to examine each second of bilateral upper limb activity throughout a single day.

Quality of life will be indexed using the Stroke Impact Scale (SIS) ${ }^{33}$ and EuroQol-5 dimension-3 level (EQ-5D-3L). ${ }^{34}$ The SIS is a multidimensional self-report measure of health status that includes 59 items to assess strength, hand function, activities of daily living, mobility, communication, emotion, memory, thinking and participation. EQ-5D, a brief measure of health-related quality of life, was added to the protocol following recommendation from the Stroke Rehabilitation and Recovery Roundtable. ${ }^{21}$ We will use the three-level option that has five domains on all participants post September 2017.

Stroke survivor perception of upper limb recovery will be examined using the Canadian Occupational Performance Measure ${ }^{35}$ Global Rating of Change scale ${ }^{36}$ and semistructured interviews. Semistructured interviews focus on three questions: (1) What is meaningful recovery to you; (2) Do you believe you have achieved meaningful recovery (yes/no, why); and (3) Do you still have hope for meaningful recovery (yes/no, why). These questions reflect themes related to recovery that were identified by previous qualitative research of individuals with upper limb impairment after stroke. ${ }^{37} 38$

Upper limb activity will also be measured using the Action Research Arm Test (ARAT). The ARAT consists of 19 items grouped into four subscales: grasp, grip, pinch and gross movement. Each subscale is ordered hierarchically, with a minimum score of 0 and a maximum score of 57. ARAT has been recommended as a common data element since commencement of this study ${ }^{21}$ and will be collected on all participants post publication of the recommendations paper (September 2017). Data will be used to model recovery trajectory on the available subset only.

Non-motor outcomes: At each time point, we will also assess non-motor outcomes which may be used as covariates in subsequent analyses. Sensation will be indexed using the light touch portion of the FM-UL assessment, cognition using the Montreal Cognitive Assessment, fatigue using the Fatigue Assessment Scale, depression using Patient Health Questionnaire-9 (PHQ-9).

\section{Data analysis and management}

Sample size

Statistical power was based on which potential biomarker(s) at $<2-4$ weeks differentiates trajectory of recovery at 12 months poststroke. Past data informed our calculation. ${ }^{22}{ }^{26}$ Using $\mathrm{R},{ }^{39}{ }^{40}$ we determined the $\mathrm{n}$ /group necessary to achieve $80 \%$ power to detect a standardised difference in slope between the group defined as 'biomarker positive' (eg, MEP+) versus 'biomarker negative' (eg, MEP-) slopes in a multilevel model with time nested within participants. ${ }^{41}$ Given the exploratory nature, the analysis plan is flexible, designed to 
Table 1 Data collected at each assessment

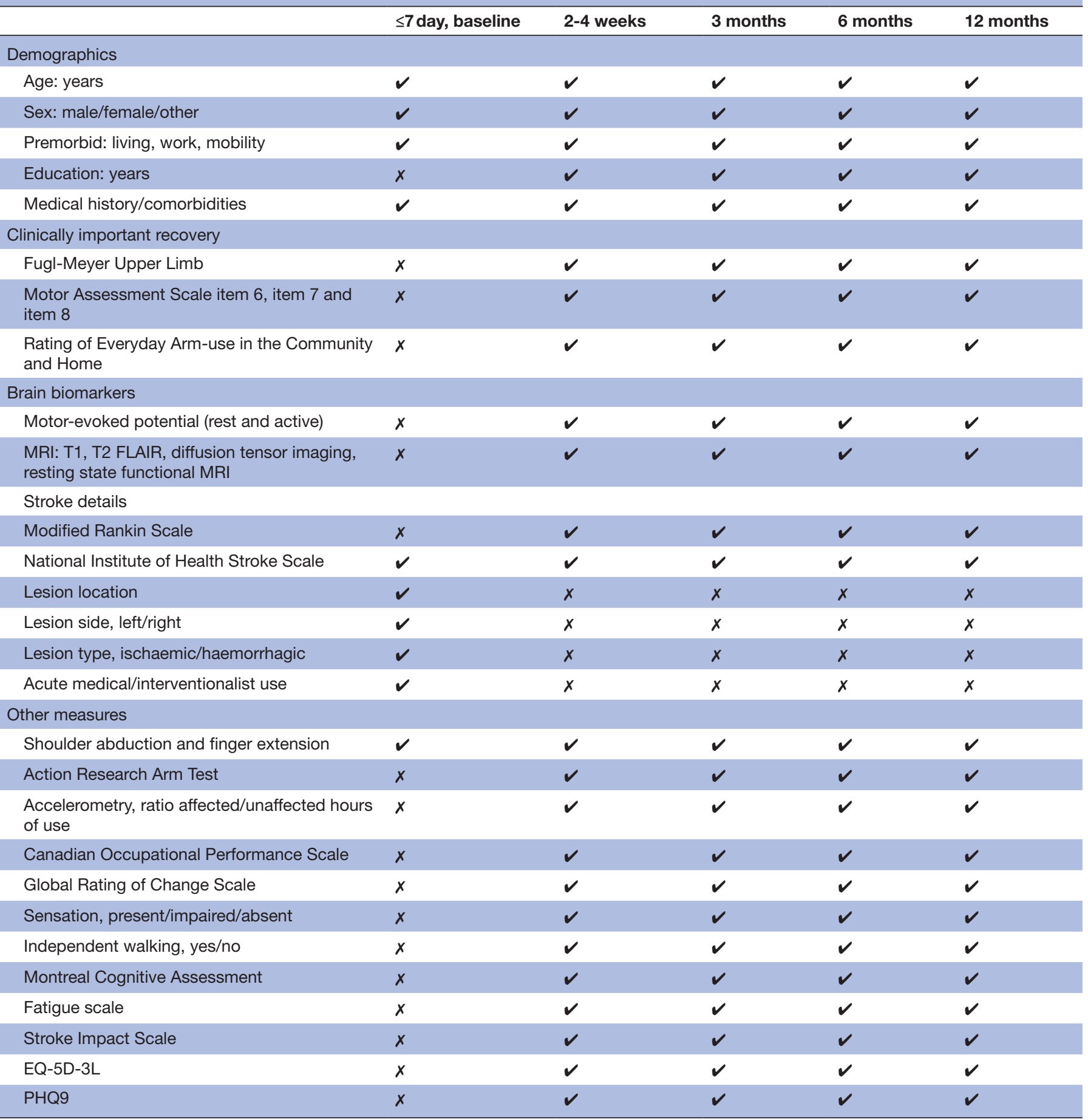

EQ-5D-3L, EuroQol five dimension three level; FLAIR, FLuid-Attenuated Inversion Recovery; PHQ-9, Patient Health Questionnaire-9.

test several different potential biomarkers, and the false positive rate is adjusted accordingly, alpha $=0.01$. For any given biomarker, assuming standardised difference in slopes is 0.245 between the 'biomarker positive' versus 'biomarker negative' groups, 32 subjects/group yields $80 \%$ power for a group by time interaction (alpha $=0.01$, average correlation between time points=0.6). We accounted for $10 \%$ loss due to death or relocation, and $10 \%$ inflation for non-compliance; thus, we are targeting recruitment of 78 participants.
Data management

All clinical measures will be initially collected on paper data forms (and securely stored) and subsequently entered into a tailored spreadsheet. All data will be confirmed by an independent rater (using the original hard copies as a reference) prior to statistical analysis. All TMS and MRI data are collected as electronic files that will be downloaded from central servers onto local servers (and securely stored). 
Analysis of outcomes

\section{Primary aim}

Identify biomarker(s) of brain structure and/or function

We will use a logistic mixed-effects regression to model the probability of attaining clinically important change as a function of time and biomarker/s. Time will be modelled as the precise number of days poststroke for each person. The primary biomarkers of interest, informed by the literature, include time point 1 MEP status, CST FA AI and CG regional FA. In secondary analyses, we will combine sets of biomarkers to determine if we can better discriminate clinically important recovery achievement. To accommodate multiple comparisons, alpha $=0.01$ will indicate significance. We will control for age, sex, stroke severity (NIHSS) and baseline SAFE score.

\section{Secondary aims}

Describe from the stroke survivor's perspective what is meaningful recovery and when it is achieved

We will explore alignment between achievement of clinically meaningful recovery (yes/no, per proposed metric) and stroke survivor's own perception of recovery; all of which are collected at every time point of this study. Using mixed-effect regression, we will model the relationship between participants' perceptions of their recovery (as the dependent variable) and clinical measures of motor function (eg, FM-UL, MAS6, REACH, SIS, EQ-5D), controlling for static variables (eg, age at stroke admission, sex). Similarly, we will model the relationship between perceptions of recovery and more ecological measures of use (eg, accelerometry-based measures of use and use asymmetry), controlling for the same covariates.

\section{Characterise the trajectory of motor recovery, and brain structure and function}

A multilevel analysis with time nested within participants using linear mixed-effect regression modelling will be run for each a priori measure of recovery (eg, FM-UL, MAS6, REACH, SIS, EQ-5D), brain structure (eg, CST FA AI) and function (eg, MEP status). These analyses will treat each outcome continuously and control for the same covariates as our primary analyses. A major goal of these exploratory analyses is to identify the best approach to model recovery over time (eg, curvilinear models vs truly non-linear models, like a negative exponential function) and identify the biomarkers that explain the most variance in these trajectories at each of the time points investigated. This approach will allow us to explore both early and late recovery phases.

\section{ETHICS AND DISSEMINATION}

This study is registered at clinicaltrials.gov (NCT02464085). The Strengthening the Reporting of Observational Studies in Epidemiology statement will guide study conduct and reporting. ${ }^{42}$ The results of this study will be disseminated via peer-reviewed journals; local, national and international meetings and conferences; and investigator/institutional websites and associated media departments.

\section{PATIENT AND PUBLIC INVOLVEMENT}

Patients and public were not involved in the study.

\section{DISCUSSION}

Our study evaluates clinical and brain measures, and will provide the most comprehensive characterisation of the long-term trajectory of motor recovery in the subgroup of individuals with severe upper limb paresis early after stroke. This study has the potential to identify potential biomarkers that explain who will show clinically important recovery, as well as demonstrate how this relates to the stroke survivor's own perception of recovery. Identifying biomarkers that are reliably associated with meaningful recovery will inform individualised treatment decisions, stratification for future clinical trials and identification of novel treatment targets.

\section{Author affiliations}

${ }^{1}$ Brain Behaviour Laboratory, Physical Therapy, University of British Columbia, Vancouver, BC, Canada

${ }^{2}$ AVERT Early Rehabilitation Research Group, Stroke Theme, Florey Institute of Neuroscience and Mental Health, Melbourne, Victoria, Australia

${ }^{3} \mathrm{NHMRC}$ Centre of Research Excellence in Stroke Rehabilitation and Brain Recovery, Melbourne, Victoria, Australia

${ }^{4}$ Department of Health, Kinesiology, Recreation; Department of Physical Therapy and Athletic Training, University of Utah, Utah, Salt Lake City, USA

${ }^{5}$ Physical Therapy, Occupational Therapy, and Neurology, Washington University School of Medicine, St Louis, Missouri, USA

Acknowledgements We thank the Physiotherapy and Occupational Therapy departments at Vancouver General Hospital, GF Strong Rehabilitation Centre and Holy Family Hospital for their support with recruitment for this study, as well as study personnel that assist with recruitment and assessments including (in alphabetical order) Angela Auriat, Janice Eng, Jennifer K Ferris, Samantha J Feldman, Chihya Hung, Tara Klassen, Tamara Koren, Beverley Larssen, Moira McPeake, Jason L Neva, Sue Peters, Lisa Simpson, Asha Toner and Katherine White KSH received support from the National Health and Medical Research Council (NHMRC) of Australia (GNT1088449), Michael Smith Foundation for Health Research (15980) and the Canadian Heart and Stroke Foundation; JB receives support from NHMRC of Australia (1058635); CEL receives support from NIH (R01 HD068290); and LAB received support from the Canada Research Chairs (Cl-SCH-01796).

Contributors $\mathrm{KSH}$ contributed intellectually and led writing the manuscript. $\mathrm{LAB}, \mathrm{JB}, \mathrm{CEL}$ and KRL contributed intellectually and edited the manuscript. KRL completed sample size calculation and statistical analysis plan.

Funding This work is supported by a Canadian Institutes of Health Research (CIHR) project grant (PJT-153330, 2017-2021) awarded to LAB, KSH, JB, CEL and KRL; and a Jakeway Foundation Grant for project start-up (2016) awarded to $L A B$ and KSH.

Competing interests None declared.

Patient consent Not required.

Ethics approval Universityof British Columbia and Vancouver Coastal Health Research Institute grantedethical approval (H15-00083).

Provenance and peer review Not commissioned; peer reviewed for ethical and funding approval prior to submission.

Open access This is an open access article distributed in accordance with the Creative Commons Attribution Non Commercial (CC BY-NC 4.0) license, which permits others to distribute, remix, adapt, build upon this work non-commercially, 
and license their derivative works on different terms, provided the original work is properly cited, appropriate credit is given, any changes made indicated, and the use is non-commercial. See: http://creativecommons.org/licenses/by-nc/4.0/.

\section{REFERENCES}

1. Nakayama H, Jørgensen HS, Raaschou HO, et al. Recovery of upper extremity function in stroke patients: the copenhagen stroke study. Arch Phys Med Rehabil 1994;75:394-8.

2. Winters $\mathrm{C}$, Heymans MW, van Wegen EE, et al. How to design clinical rehabilitation trials for the upper paretic limb early post stroke? Trials 2016;17:468.

3. Byblow WD, Stinear CM, Barber PA, et al. Proportional recovery after stroke depends on corticomotor integrity. Ann Neurol 2015;78:848-59.

4. Boyd LA, Hayward KS, Ward NS, et al. Biomarkers of stroke recovery: consensus-based core recommendations from the stroke recovery and rehabilitation roundtable. Int J Stroke 2017;12:480-93.

5. Hayward KS, Schmidt J, Lohse KR, et al. Are we armed with the right data? Pooled individual data review of biomarkers in people with severe upper limb impairment after stroke. Neuroimage Clin 2017;13:310-9.

6. Stinear CM, Byblow WD, Ackerley SJ, et al. PREP2: A biomarkerbased algorithm for predicting upper limb function after stroke. Ann Clin Transl Neurol 2017;4:811-20.

7. Zarahn E, Alon L, Ryan SL, et al. Prediction of motor recovery using initial impairment and fMRI $48 \mathrm{~h}$ poststroke. Cereb Cortex 2011;21:2712-21.

8. Hendricks HT, Pasman JW, Merx JL, et al. Analysis of recovery processes after stroke by means of transcranial magnetic stimulation. J Clin Neurophysiol 2003;20:188-95.

9. van Kuijk AA, Pasman JW, Hendricks HT, et al. Predicting hand motor recovery in severe stroke: the role of motor evoked potentials in relation to early clinical assessment. Neurorehabil Neural Repair 2009;23:45-51.

10. Burke Quinlan E, Dodakian L, See J, et al. Neural function, injury, and stroke subtype predict treatment gains after stroke. Ann Neurol 2015;77:132-45.

11. Feng W, Wang J, Chhatbar PY, et al. Corticospinal tract lesion load: an imaging biomarker for stroke motor outcomes. Ann Neurol 2015;78:860-70

12. Bigourdan A, Munsch $F$, Coupé $P$, et al. Early fiber number ratio is a surrogate of corticospinal tract integrity and predicts motor recovery after stroke. Stroke 2016;47:1053-9.

13. Hayward KS, Ferris J, Lohse KR, et al. Biomarkers of upper limb outcome post-stroke: One-size-fits-all? International Journal of Stroke. Conference Proceedings 2018.

14. Hayward KS, Neva JL, Mang CS, et al. Interhemispheric pathways are important for motor outcome in individuals with chronic and severe upper limb impairment post stroke. Neural Plast 2017;2017:1-12

15. McPherson JG, Chen A, Ellis MD, et al. Progressive recruitment of contralesional cortico-reticulospinal pathways drives motor impairment post stroke. J Physiol 2018;596:1211-25.

16. Quinlan EB, Dodakian L, See J, et al. Biomarkers of rehabilitation therapy vary according to stroke severity. Neural Plast 2018;2018:1-8

17. Cortes JC, Goldsmith J, Harran MD, et al. A Short and distinct time window for recovery of arm motor control early after stroke revealed with a global measure of trajectory kinematics. Neurorehabil Neural Repair 2017:31:552-60.

18. Duncan PW, Goldstein LB, Matchar D, et al. Measurement of motor recovery after stroke. Outcome assessment and sample size requirements. Stroke 1992;23:1084-9.

19. Bernhardt J, Hayward KS, Kwakkel G, et al. Agreed definitions and a shared vision for new standards in stroke recovery research: the stroke recovery and rehabilitation roundtable taskforce. Int J Stroke 2017;12:444-50.

20. Ganesh A, Gutnikov SA, Rothwell PM. Late functional improvement after lacunar stroke: a population-based study. J Neurol Neurosurg Psychiatry 2018:jnnp-2018-318434.
21. Kwakkel G, Lannin NA, Borschmann K, et al. Standardized measurement of sensorimotor recovery in stroke trials: consensusbased core recommendations from the Stroke Recovery and Rehabilitation Roundtable. Int J Stroke 2017;12:451-61.

22. Lang CE, Edwards DF, Birkenmeier RL, et al. Estimating minimal clinically important differences of upper-extremity measures early after stroke. Arch Phys Med Rehabil 2008;89:1693-700.

23. Platz T, Pinkowski C, van Wijck F, et al. Reliability and validity of arm function assessment with standardized guidelines for the fugl-meyer test, action research arm test and box and block test: a multicentre study. Clin Rehabil 2005;19:404-11.

24. Duncan PW, Propst M, Nelson SG. Reliability of the Fugl-Meyer assessment of sensorimotor recovery following cerebrovascular accident. Phys Ther 1983;63:1606-10.

25. Dean C, Mackey F. Motor assessment scale scores as a measure of rehabilitation outcome following stroke. Aust $J$ Physiother 1992;38:31-5.

26. Hayward KS, Kuys SS, Barker RN, et al. Can stroke survivors with severe upper arm disability achieve a clinically important change in arm function during inpatient rehabilitation? A multicentre, prospective, observational study. NeuroRehabilitation 2014;35:773-9.

27. Simpson LA, Eng JJ, Backman CL, et al. Rating of Everyday ArmUse in the Community and Home (REACH) scale for capturing affected arm-use after stroke: development, reliability, and validity. PLoS One 2013;8:e83405.

28. Mang CS, Borich MR, Brodie SM, et al. Diffusion imaging and transcranial magnetic stimulation assessment of transcallosal pathways in chronic stroke. Clin Neurophysiol 2015;126:1959-71.

29. Zabukovec JR, Boyd LA, Linsdell MA, et al. Changes in corticospinal excitability following adaptive modification to human walking. Exp Brain Res 2013;226:557-64.

30. Feldman SJ, Boyd LA, Neva JL, et al. Extraction of corticospinal tract microstructural properties in chronic stroke. J Neurosci Methods 2018;301:34-42.

31. Hofer S, Frahm J. Topography of the human corpus callosum revisited-comprehensive fiber tractography using diffusion tensor magnetic resonance imaging. Neuroimage 2006;32:989-94.

32. Bailey RR, Klaesner JW, Lang CE. Quantifying real-world upperlimb activity in nondisabled adults and adults with chronic stroke. Neurorehabil Neural Repair 2015;29:969-78.

33. Duncan PW, Wallace D, Lai SM, et al. The stroke impact scale version 2.0. Evaluation of reliability, validity, and sensitivity to change. Stroke 1999;30:2131-40.

34. Golicki D, Niewada M, Karlińska A, et al. Comparing responsiveness of the EQ-5D-5L, EQ-5D-3L and EQ VAS in stroke patients. Qual Life Res 2015;24:1555-63.

35. Law M, Baptiste S, McColl M, et al. The canadian occupationa performance measure: an outcome measure for occupational therapy. Canadian Journal of Occupational Therapy 1990;57:82-7

36. Kamper SJ, Maher CG, Mackay G. Global rating of change scales: a review of strengths and weaknesses and considerations for design. J Man Manip Ther 2009;17:163-70.

37. Barker RN, Brauer SG. Upper limb recovery after stroke: the stroke survivors' perspective. Disabil Rehabil 2005;27:1213-23.

38. Barker RN, Gill TJ, Brauer SG. Factors contributing to upper limb recovery after stroke: a survey of stroke survivors in Queensland Australia. Disabil Rehabil 2007;29:981-9

39. Donohue MC, Edland SD. longpower: power and sample size calculators for longitudinal data. $R$ package version, 2016:10-16.

40. Liu G, Liang KY. Sample size calculations for studies with correlated observations. Biometrics 1997;53:937-47.

41. Diggle PJ, Heagerty PJ, Liang K, et al. Analysis of longitudinal data. 2nd: Oxford Statistical Science Series, 2002.

42. von Elm E, Altman DG, Egger M, et al. The Strengthening the Reporting of Observational Studies in Epidemiology (STROBE) statement: guidelines for reporting observational studies. Lancet 2007;370:1453-7. 